\title{
La contribución de las tecnologías Web 2.0 a la formación de pacientes activos
}

\author{
The contribution of Web 2.0 technologies \\ to the empowerment of active patients
}

Martí Oliver-Mora ${ }^{1}$

Lupicinio Iñiguez-Rueda ${ }^{1}$

${ }^{1}$ Departamento de Psicología Social, Facultad de Psicología, Universidad Autónoma de Barcelona. Edificio B, Campus de Bellaterra. 08193 Bellaterra España.

m_oliver_m@mac.com

\begin{abstract}
The Spanish health system has recently been marked by the emergence of more active patients who are characterized as being better informed about their disease, having a more participatory attitude, wanting to have a greater influence in making decisions about their health and asserting their rights as patients. Therefore, this article aims to report on how the introduction of Web 2.0 technologies can contribute to the empowering of more active patients. To achieve this, 14 semi-structured interviews were conducted with patients and representatives of patient associations who have used Web 2.0 technologies to interact with other patients or to communicate with health professionals. From the results obtained, we highlight the fact that Web 2.0 technologies provide greater access to health-related information, improve communication between patients and health professionals, and enable the creation of new spaces of interaction among patients. All of the facts above contribute to the formation of a more active role on the part of patients.
\end{abstract}

Key words Public health, Means of communication, Needs and demands of health services, Dissemination of innovation, Qualitative research
Resumen El sistema sanitario español ha estado marcado recientemente por la emergencia de pacientes más activos que se caracterizan por estar mejor informados sobre su enfermedad, por disponer de una actitud más participativa, por querer incidir en mayor medida en la toma de decisiones sobre su salud y por reivindicar sus derechos como pacientes. Tomando este punto de partida, el presente artículo tiene como objetivo dar cuenta de cómo la introducción de las tecnologías Web 2.0 puede contribuir a la configuración de un nuevo modelo de pacientes más activos. Para ello, hemos realizado 14 entrevistas semi-estructuradas a pacientes y a representantes de asociaciones de pacientes que hayan utilizado las tecnologías Web 2.0 para relacionarse con otros pacientes o para comunicarse con sus profesionales de la salud de referencia. Entre los resultados obtenidos, destacamos que las tecnologías Web 2.0 proporcionan un mayor acceso a información relacionada con la salud, mejoran la comunicación entre los pacientes y sus profesionales de referencia, y permiten la creación de nuevos espacios de interacción entre pacientes. Todo ello contribuye a la formación de un rol más activo por parte de los pacientes.

Palabras clave Salud pública, Medios de comunicación, Necesidades y demandas de servicios de salud, Difusión de innovaciones, Investigación cualitativa 


\section{Introducción}

Desde hace varias décadas, la palabra paciente se ha consolidado en España como el término de referencia para denominar a aquellas personas que padecen una enfermedad y se hallan bajo atención médica1. Sin embargo, en algunos entornos como el anglosajón, pero progresivamente también en España, la palabra paciente se nos ha quedado corta. El rol activo que asumen muchas personas en relación a la condición de paciente nos obliga a acompañar este término con nuevos adjetivos, como inquieto, crítico, participativo, experto, y un largo etcétera ${ }^{2-4}$.

En términos generales, podemos decir que el nuevo paciente activo se caracteriza por adoptar una actitud más participativa en su relación con el sistema sanitario, por estar mejor informado sobre su enfermedad, por tener una mayor voluntad de decidir sobre su salud y por tomar conciencia y reclamar sus derechos como paciente ${ }^{3}$. En este sentido, existe una larga bibliografía entorno a este nuevo modelo de paciente, lo cual refleja la voluntad de los profesionales de la salud de comprender los cambios de su entorno y así poder atender mejor sus demandas 5 . Sin embargo, más importante aún en la emergencia y en el impacto de este nuevo modelo de paciente ha sido el papel de la sociedad civil, agrupada principalmente en asociaciones de pacientes, ya que ha jugado un rol fundamental al trasladar sus demandas y necesidades hacia la consecución de un nuevo modelo asistencial.

La experiencia más significativa impulsada en España en este sentido fueron las jornadas organizadas por la Fundación Biblioteca Josep Laporte en mayo de 2003. Estas jornadas reunieron a profesionales de la salud, a representantes de organizaciones y asociaciones de pacientes, y a usuarios de diferentes lugares de España. El resultado fue la denominada Declaración de Barcelona de las Asociaciones de Pacientes, que contiene el Decálogo de los Pacientes (Cuadro 1) donde se estipulan las principales necesidades de los pacientes en relación a los servicios asistenciales prestados por la sanidad pública española:

Como vemos, los diez puntos del decálogo pueden agruparse en las cuatro grandes características que definen al nuevo paciente activo mencionadas anteriormente: una actitud más participativa, mejor información sobre la enfermedad, mayor poder en la toma de decisiones sobre la salud y reivindicación de sus derechos.

A pesar de los años transcurridos desde la $D e$ claración de Barcelona de las Asociaciones de Pa-
Cuadro 1. Decálogo de los pacientes.

\begin{tabular}{l} 
Información de calidad contrastada respetando la \\
pluralidad de las fuentes \\
\hline Decisiones centradas en el paciente \\
\hline $\begin{array}{l}\text { Respeto a los valores y a la autonomía del paciente } \\
\text { informado }\end{array}$ \\
\hline $\begin{array}{l}\text { Relación médico-paciente basada en el respeto y la } \\
\text { confianza mutua }\end{array}$ \\
\hline $\begin{array}{l}\text { Formación y entrenamiento específico en } \\
\text { habilidades de comunicación para profesionales }\end{array}$ \\
\hline $\begin{array}{l}\text { Participación de los pacientes en la determinación } \\
\text { de prioridades de asistencia sanitaria }\end{array}$ \\
\hline Democratización formal de las decisiones sanitarias \\
\hline $\begin{array}{l}\text { Reconocimiento de las organizaciones de pacientes } \\
\text { como agentes de política sanitaria }\end{array}$ \\
\hline $\begin{array}{l}\text { Mejora del conocimiento que tienen los pacientes } \\
\text { sobre sus derechos básicos }\end{array}$ \\
\hline $\begin{array}{l}\text { Garantía de cumplimiento de los derechos básicos } \\
\text { de los pacientes }\end{array}$ \\
Fuente: Zapater .
\end{tabular}

Fuente: Zapater ${ }^{3}$.

cientes, muchas de las necesidades sociales expresadas por los pacientes en este manifiesto siguen igual de vigentes a día de hoy. Por una parte, las administraciones competentes no han impulsado las políticas públicas necesarias para introducir los diez puntos del decálogo entre los pilares de la sanidad pública española; y por otra parte, los pacientes activos siguen siendo muy minoritarios en términos cuantitativos, siendo el perfil del paciente pasivo o sobreprotegido el modelo de paciente predominante 3 .

De todos modos $-y$ ante la ausencia de grandes mediadas institucionales a favor de fomentar pacientes más activos -, los avances tecnológicos de estos últimos años en el campo de las tecnologías de la información y la comunicación (TIC), y especialmente en el ámbito de las tecnologías Web 2.0, han puesto sobre la mesa nuevas herramientas que pueden contribuir a la consecución de los objetivos fijados en la Declaración de Barcelona. Las tecnologías Web 2.0, es decir, las que permiten una mayor interacción entre las personas (redes sociales, comunidades virtuales, etc.), están jugando un papel cada vez más importante en nuestras vidas, y como consecuencia, también en la de los pacientes. Así, y como veremos más adelante, estos aprovechan las posibilidades que ofrecen dichas herramientas para buscar información sobre su enfermedad, para relacionarse con otros pacientes, e incluso para hacer consul- 
tas telemáticas a sus profesionales sanitarios de referencia.

Por su parte, los profesionales del sistema sanitario español están jugando un rol cada vez más importante en el impulso de experiencias innovadoras relacionadas con la aplicación de las tecnologías Web 2.0 al ámbito de la salud. En su gran mayoría no se trata de grandes proyectos institucionales, sino de iniciativas impulsadas desde abajo hacia arriba por parte de determinados Equipos de Atención Primaria, y que por lo tanto no benefician a una gran cantidad de pacientes. Sin embargo, estos profesionales de la salud han sabido recoger las demandas de los pacientes utilizando las tecnologías Web 2.0 para fomentar un rol más activo por parte de los pacientes $^{6}$

En este sentido, la creciente aplicación de tecnologías cada vez más sofisticadas al ámbito de la salud ha dado lugar a conceptos como "eSalud", "telemedicina" o "Salud 2.0"7-10. En esta investigación hemos ubicado nuestro campo de estudio en el ámbito definido por este último concepto, que hace referencia a la aplicación de las tecnológicas Web 2.0 al ámbito de la salud. En un artículo anterior centramos nuestra atención en algunas de las experiencias innovadoras en el uso de las tecnologías Web 2.0 impulsadas por los profesionales de la atención primaria en España que pueden contribuir a mejorar la prestación de los servicios públicos ${ }^{6}$. En esta ocasión, sin embargo, nuestro objetivo consiste en analizar la visión de los pacientes en España acerca de las potencialidades de las tecnologías Web 2.0 para dar respuesta a sus necesidades, y dar cuenta de cómo la introducción de estas tecnologías puede contribuir a la formación de pacientes más activos.

\section{Métodos}

Para analizar la manera en que las tecnologías Web 2.0 pueden contribuir a configurar un modelo de pacientes más activos, hemos realizado 14 entrevistas semi-estructuradas a pacientes o a representantes de asociaciones de pacientes que hayan utilizado las tecnologías Web 2.0 en el ámbito de la salud. En este sentido, hemos seleccionado a tres perfiles diferenciados de pacientes o representantes de asociaciones de pacientes, que responden a diferentes formas de relacionarse con estas herramientas.

En primer lugar, hemos entrevistado a pacientes que hayan participado en experiencias pioneras en la aplicación de las tecnologías Web 2.0 en el ámbito de la salud en España. Concretamente, hemos entrevistado a pacientes vinculados a tres proyectos de Salud 2.0: Kronikoen Sarea en el País Vasco, Canal Pacient en Cataluña y la Escuela de Pacientes en Andalucía. Estas tres experiencias fueron seleccionadas en una fase previa de este proyecto de investigación, consistente en la identificación de experiencias innovadoras que fueran capaces de mejorar la prestación de servicios públicos mediante la implementación de las tecnologías Web 2.0 en el ámbito de la salud ${ }^{6}$. El objetivo de entrevistar a pacientes de este primer perfil era contar con el discurso de personas implicadas en grandes proyectos de Salud 2.0, ya que se trata de experiencias que a día de hoy aún resultan minoritarias, pero que pueden extenderse e implicar a un mayor número de personas en el futuro.

En segundo lugar, hemos entrevistado a pacientes que sin participar en grandes proyectos de Salud 2.0, utilizan las tecnologías Web 2.0 generalistas en su día a día con fines sanitarios. Se trata de pacientes que utilizan herramientas como Facebook, Twitter o Whatsapp, entre otras, ya sea para buscar o compartir información sobre su enfermedad, ya sea para relacionarse con otros pacientes para lograr un mayor apoyo mutuo. El objetivo de entrevistar a pacientes de este segundo perfil era acercarnos a unos usos más habituales de las tecnologías Web 2.0 y que puede representar a un mayor número de pacientes en términos cuantitativos.

Finalmente, hemos entrevistado a representantes de asociaciones de pacientes que han reflexionado en torno a las potencialidades de las tecnologías Web 2.0 aplicadas al ámbito sanitario. Contar con este último perfil nos ha acercado a los discursos de personas expertas que trabajan fomentando el buen uso de estas herramientas por parte de los pacientes, a través de los diferentes cursos o actividades que realizan desde las asociaciones.

Dada la gran dispersión geográfica de las personas entrevistadas, hemos intercalado la realización de entrevistas presenciales con la realización de entrevistas on-line mediante el programa Skype. Esto nos ha permitido entrevistar a pacientes de diferentes regiones de España, y por lo tanto, implicados en diversos proyectos de Salud 2.0 o que participan en asociaciones de pacientes de diferentes ámbitos territoriales.

Todas las entrevistas tuvieron una duración de entre 30 minutos y 1 hora, y se estructuraron en torno a tres grandes apartados: I. Pacientes y Salud 2.0; II. Necesidades de los pacientes; y III. 
Necesidades de los pacientes y Salud 2.0. El primer apartado hace referencia a la introducción de las tecnologías Web 2.0 en el ámbito sanitario (utilización por parte de los pacientes, profesionales de la salud, etc.). El segundo apartado, por su parte, tiene como objetivo identificar las principales necesidades de la persona entrevistada en relación a su enfermedad. En el caso de los representantes de las asociaciones de pacientes, se abordan las necesidades relacionadas con la enfermedad de los pacientes asociados. Finalmente, el último apartado pone en relación el contenido abordado en los dos apartados precedentes intentando establecer la manera en que las tecnologías Web 2.0 pueden dar respuesta a las necesidades manifestadas por los pacientes.

Una vez realizadas las entrevistas, las hemos transcrito con el fin de poder analizar su contenido más detalladamente. Este análisis lo hemos llevado a cabo mediante el programa informático Atlas.ti, especializado en el análisis cualitativo de texto. Hemos analizado las entrevistas aplicando la técnica del análisis de contenido ${ }^{11}$. Esta técnica permite sistematizar la información obtenida en las entrevistas mediante la formulación de inferencias que remitan a un contexto específico, ya sea textual (contenido explícito de las transcripciones), ya sea social (características del enunciante, circunstancias espaciales y temporales en las que se produce, etc.). En definitiva, esta técnica consiste en agrupar el texto que está siendo analizado en unidades con un valor temático compartido, para posteriormente agrupar estas unidades en categorías de mayor dimensión, construidas por analogía según los objetivos de investigación establecidos.

En nuestro caso, hemos definido 19 unidades temáticas relacionadas con el contenido de las entrevistas. Algunas de estas unidades temáticas hacen referencia a necesidades concretas expresadas por parte de las personas entrevistadas, mientras que otras hacen referencia a la experiencia de la persona entrevistada en el uso de las tecnologías Web 2.0, a las reticencias expresadas por algunas de estas personas a utilizar en mayor medida este tipo de tecnologías, o a su trayectoria política y asociativa.

Estas unidades temáticas fueron posteriormente agrupadas en categorías de mayor dimensión según su afinidad de contenido y según los objetivos de investigación establecidos. Fue mediante esta categorización ulterior que pudimos identificar y delimitar las principales necesidades expresadas por las personas entrevistadas que pueden ser resueltas, o parcialmente resueltas, con el uso de tecnologías Web 2.0. En este sentido, la ordenación de los resultados que se presentan a continuación responde a las tres necesidades principales identificadas durante este proceso de análisis: un mayor acceso a información sanitaria, una mejor comunicación con los profesionales sanitarios de referencia y un mayor apoyo mutuo entre pacientes.

\section{Resultados}

\section{Acceso a la información sanitaria}

Hemos identificado el mayor acceso a la información sanitaria como una de las principales necesidades expresadas por los pacientes y que puede ser cubierta, o parcialmente cubierta, con el uso de las tecnologías Web 2.0. Como en otros ámbitos de nuestra vida cotidiana, internet está ocupando un lugar cada vez más importante para resolver las dudas e inquietudes de los pacientes sobre sus enfermedades. Sin embargo, cabe distinguir diferentes momentos e intensidades en el uso de internet como fuente de información por parte de los pacientes.

El primer momento, y seguramente el más crítico, es el inmediatamente posterior al diagnóstico, en que las dudas son más generales, los conocimientos más escasos, y por lo tanto, el momento en que las búsquedas están peor dirigidas. En palabras del representante de una asociación de pacientes:

En el momento en el que más gente entra a consultar es en el momento del diagnóstico, es en el momento en que tú entras a mirar, y la gente nos llega bastante asustada. De hecho, nosotros el primer consejo que damos es que no entren de manera indiscriminada a buscar, sino que vayan a fuentes fiables, y por esto nuestra página web tiene bastante contenido de información, porque queremos ser un poco una referencia en este sentido (Carlos).

En algunas ocasiones, esta búsqueda indiscriminada es una respuesta a la escasa información sobre la enfermedad proporcionada por parte de los profesionales de la salud justo después del diagnóstico. En cualquier caso, la existencia de internet ha facilitado el acceso a información sanitaria por parte de los pacientes, de manera que el hecho de que el sistema sanitario no satisfaga las necesidades informativas de los pacientes puede tener efectos negativos, dando lugar a búsquedas más o menos aleatorias en internet, $\mathrm{o}$ como comentan algunos pacientes, "consultando al Dr. Google". 
Otra práctica relacionada con la necesidad de obtener mayor información sanitaria por parte de los pacientes, y que generalmente se lleva a cabo más adelante en el tiempo, hace referencia al hecho de establecer contacto con otros pacientes que se encuentren en una situación similar. En este sentido, y en contraposición a la información más técnica (relegada en mayor medida a los profesionales sanitarios), los otros pacientes son percibidos como la fuente de información más eficaz sobre las cuestiones prácticas del día a día que hacen referencia a la enfermedad:

Yo no quiero menospreciar ni muchísimo menos el trabajo de los médicos, pero el trabajo de los médicos en una enfermedad que tiene tanta implicación social es muy importante a nivel de diagnóstico y de tratamiento en caso de una evolución de la enfermedad, pero en el día a día se queda muy coja, porque tu sales de una consulta diciendo "tu hijo no puede tomar ni leche, ni huevos, ni frutos secos", pero ¿cómo encuentras los productos? ¿Qué son las trazas? ¿Mi hijo va a tolerar las trazas? Todo esto al final es entre pacientes, $y$ asociaciones (Laura).

Es precisamente en esta voluntad de obtener mayor información relacionada con la enfermedad a través de la relación con otros pacientes que las tecnologías Web 2.0 resultan especialmente útiles. La herramienta más utilizada en este sentido es sin duda Facebook, y concretamente, los grupos de Facebook que ponen en contacto a personas con una misma enfermedad. Estos grupos ofrecen características muy variadas: pueden estar gestionados por organizaciones con una cierta estructura o simplemente por personas individuales, pueden estar centrados en el carácter vivencial de la enfermedad o tener un carácter más informativo, y pueden estar más dirigidos por sus impulsores o disponer de una participación más horizontal. Sin embargo, y más allá de las características particulares de cada grupo, la mayor ventaja que ofrecen para los pacientes radica en su elevada participación, lo cual facilita resolver las dudas con rapidez y poder mantener relaciones más fluidas con otros pacientes:

[...] luego ya empezaron a surgir grupos muy determinados que son grupos muy cerrados de Facebook a los que tú pides permiso, el administrador te da permiso, son grupos cerrados a nivel internacional, muchos de Sudamérica, España y Sudamérica sobre todo, en los que hay "padres de niños con alergia a la leche", "padres de niños con alergia a leche y huevos", etc. Puede haber seis o siete ahora mismo, $y$ en esos grupos hay un intercambio rapidísimo de información, porque ahora con los móviles... (Laura).
Sin embargo, esta elevada participación también da lugar a un mayor desconocimiento entre participantes, y por lo tanto, a una mayor desconfianza en la información proporcionada. Mención a parte merecen los grupo de Facebook más reducidos, normalmente con carácter cerrado y gestionados mayoritariamente por asociaciones de pacientes. En estos grupos los pacientes suelen tener relaciones más estrechas, incluso a nivel presencial, y el hecho de que estén gestionados por una organización de su confianza hace más creíble para los pacientes la información que proporcionan.

Por otra parte, también los grupos de Whatsapp están teniendo un rol cada vez más protagonista en la transmisión de información entre pacientes. Así, y a semejanza de los grupos de Facebook, algunas asociaciones de pacientes disponen de grupos de Whatsapp a través de los cuales realizan un intercambio de información más fluido y esporádico. Estos grupos pueden incluso verse reforzados con la entrada de otros pacientes invitados por alguno de sus miembros, pero desconocidos por los demás participantes, convirtiéndose de este modo en auténticos foros donde interactúan pacientes de ámbitos territoriales muy diversos.

\section{Comunicación con los profesionales de la salud}

En las entrevistas realizadas hemos identificado la necesidad por parte de los pacientes de mantener una mejor comunicación con sus profesionales sanitarios de referencia. Las causas que generan esta necesidad pueden ser muy variadas, como por ejemplo el escaso tiempo que dedican los profesionales de la salud a cada consulta, las dificultades por parte de los pacientes de entender las indicaciones que los profesionales sanitarios les proporcionan en la consulta, o el llamado "síndrome de la bata blanca", es decir, el estrés involuntario que provoca el entorno sanitario sobre algunos pacientes.

Algunas de estas cuestiones pueden verse reflejadas en el tipo de entradas que realiza una de las personas entrevistadas en su blog de internet:

$Y$ lo que cuento en el blog básicamente son mis experiencias personales, desde lo que me cuesta subir unas escaleras mecánicas, a lo que es tener miedo de ir al médico, mi experiencia de que tengo que ir acompañada al médico porque voy y luego los dos tenemos versiones totalmente diferentes de lo mismo (Marta).

La tecnología Web 2.0 más utilizada para mejorar la comunicación entre pacientes y profesio- 
nales de la salud son los consultorios virtuales. Esta herramienta, cada vez más extendida en el sistema sanitario español, consiste en una plataforma virtual a través de la cual los pacientes pueden realizar consultas a sus profesionales sanitarios de referencia a través de un correo electrónico encriptado. En muchas ocasiones permiten adjuntar documentos, se sincronizan automáticamente con el historial clínico del paciente, y en las versiones más sofisticadas incluso permiten la realización de consultas mediante video-conferencia.

Algunos de los beneficios expresados por los pacientes que hemos entrevistado y que han utilizado este tipo de herramientas incluyen el ahorro del tiempo y de la energía que implica realizar una consulta presencial cuando el motivo de la consulta puede solucionarse telemáticamente (gestionar la receta electrónica, consultar los resultados de un análisis, gestionar las bajas laborales, etc.), poder realizar las consultas en cualquier momento del día independientemente de los horarios de consulta presencial de los profesionales de la salud de referencia, resolver dudas sobre las indicaciones señaladas por los profesionales de la salud en la consulta, y en definitiva, disponer de una relación más fluida con los profesionales de referencia:

¿Dirías que ha cambiado tu relación con tu médico y con tu enfermera de referencia por el hecho de utilizar esta herramienta? Sí, totalmente, totalmente, porque no es lo mismo un paciente que lo tengas que atender todos los días, y verle la cara todos los días, que ya es tedioso, con dolores como los míos, porque ahora por ejemplo tengo un dolor en el cuádriceps y que no sé de qué es, y el siguiente que tengo dolor de garganta, o tengo tos, y no es lo mismo mandárselo por escrito, así que ha mejorado mi relación con el médico, sí. De todas formas, cuando él no lo ve claro, me dice 'ven', o cuando lo lee, antes de que me llegue a mí la información, me llama por teléfono y me dice 'ven' (María).

Sin embargo, la mejora de la comunicación entre pacientes y profesionales de la salud no viene únicamente determinada por el hecho de poner a su alcance nuevas soluciones tecnológicas como los consultorios virtuales, sino que esta comunicación requiere al mismo tiempo una mayor implicación en la enfermedad por parte de los pacientes. En este sentido, la contribución de las tecnologías Web 2.0 a la formación de pacientes más informados -como hemos visto en el apartado anterior- también facilita indirectamente una mejor comunicación entre pacientes y profesionales de la salud en la consulta:

Hubo un momento de mi vida que yo pensaba, como todo el mundo, el no saber, la ignorancia, yo decía 'cómo voy a tener yo artritis reumatoide, esto es una enfermedad de viejos, y tengo diecinueve años y me estoy muriendo de dolor, yo tengo cáncer en los huesos, esto tiene que ser otra cosa' [...] Ahora con mi reumatóloga me llevo estupendamente, tenemos un contacto estupendo, no hay ningún problema. Discutimos los tratamientos, vemos los pros $y$ los contras, porque es mi tratamiento, es mi cuerpo, tendré yo que aceptarlo o no, no voy a ser como un borreguito a tomarme lo que me den (Lourdes).

De este modo, un paciente informado constituye un paciente que puede entender las indicaciones de sus profesionales de la salud de referencia, que puede valorar en mayor medida las particularidad de los tratamientos a los que se va a someter, y en definitiva, un paciente con una mayor capacidad de decisión. Todas estas cuestiones facilitan enormemente la comunicación entre los pacientes y los profesionales de la salud, estableciendo así una relación más fluida.

Sin embargo, el peso de facilitar una mejor comunicación entre pacientes y profesionales de la salud no recae únicamente sobre los primeros, y es que al mismo tiempo resulta imprescindible que los profesionales de la salud desarrollen una actitud más empática, ajustando sus explicaciones al nivel de comprensión de los pacientes y tomando en consideración su punto de vista.

\section{Apoyo mutuo entre pacientes}

Finalmente, el apoyo mutuo entre pacientes constituye una necesidad muy reivindicada, especialmente entre los representantes de las asociaciones que hemos entrevistado. Argumentan que el sistema sanitario está excesivamente preocupado por la recuperación física, dejando la recuperación psicológica y social en un segundo plano. Esto sucede a pesar de que el apoyo mutuo es concebido como un pilar primordial en la recuperación integral de los pacientes; así, dar respuesta a esta necesidad ha constituido durante años una de las principales funciones de muchas asociaciones de pacientes:

Hay muchas mujeres que las parejas no les ayudan nada, hay otras mujeres que les beneficia un montón, hay otras parejas que les benefician los hijos, pero todas estas preguntas, estas veces que no pueden hablarlas en las familias, las hablan en los talleres, y son las propias mujeres las que se vuelven profesoras, porque ellas mismas se implican tanto en este taller, que ellas mismas se ayudan, muchas veces las que estamos de profesoras dejamos de hablar y hablan ellas, que hablen y que se ayuden, que se cuenten (Elena). 
Más allá del trabajo realizado por parte de las asociaciones de pacientes, las tecnologías Web 2.0 han abierto nuevas posibilidades para dar respuesta a esta necesidad. Por una parte, y en contraposición a grupos presenciales de apoyo mutuo entre pacientes, internet ofrece una mayor accesibilidad a sus usuarios. Accesibilidad espacial, ya que es posible acceder a estos grupos de apoyo desde cualquier ámbito territorial, pero también accesibilidad temporal, ya que su participación no está restringida por un horario preestablecido. Por otra parte, internet ofrece la posibilidad de participar de forma pasiva y de forma anónima. Aunque pueda parecer una cuestión secundaria, esta participación pasiva y anónima posibilita que muchas personas que nunca hubieran tenido ningún tipo de contacto con grupos de apoyo se acerquen a ellos progresivamente, lo cual puede desencadenar una participación más activa en el futuro. De este modo, el uso de las tecnologías Web 2.0 ha aportado una mayor flexibilidad y transversalidad en los modos de participación en este tipo de grupos:

Cuando me presentaron lo de Kronikoen Sarea yo pensaba 'esto ya es algo que hacemos las asociaciones desde hace muchos años', el apoyo mutuo [...] Esta función que tiene la asociación es la que en cierto modo también pretende cubrir Kronikoen Sarea, con la diferencia de que Kronikoen Sarea es una plataforma abierta a cualquier tipo de patología, y no se centra en la enfermedad, sino en el estado de ánimo de las personas y en los puntos comunes que nos unen a todos, que es ese apoyo que encuentras, esa empatía que encuentras en alguien que tiene algo parecido a lo tuyo, aunque no sea médicamente igual, pero que sí que tiene muchos puntos en común y te entiendes muy bien con este tipo de personas por los sentimientos que tiene, que los compartes tu también, los has tenido en algún momento (José).

Las tecnologías utilizadas para cubrir esta necesidad varían sensiblemente con respecto a las señaladas hasta ahora. Si bien es cierto que los grupos de Facebook y los grupos de Whatsapp pueden cumplir parcialmente esta función, en general están destinados a resolver cuestiones más prácticas y a difundir información de interés, la implicación de sus participantes es menor, y los vínculos que se establecen entre ellos son más superficiales. Sin embargo, y como hemos mencionado anteriormente, también se pueden generar entornos de mayor confianza mediante estas herramientas, por ejemplo, en grupos cerrados y vinculados a organizaciones estables, como puede ser el caso de una asociación de pacientes.
Así, y aunque el peso de los grupos de Facebook y de Whatsapp en relación a esta necesidad va en aumento, siguen siendo los foros/comunidades virtuales las que generan unas relaciones más intensas entre sus participantes. Esto puede explicarse, parcialmente, por el hecho de que los foros fueron una de las primeras tecnologías interactivas que existieron en internet, y que por lo tanto, la relación entre muchos de sus participantes tiene un mayor recorrido en el tiempo:

Yo venía previamente de un foro de trastorno bipolar que se llamaba [nombre del foro], que actualmente estamos en la asociación, y yo estuve como moderadora durante muchos años en ese foro. Entonces, ese foro de pronto desapareció, y se quedó, la verdad es que se quedó gente que era necesario tener ese contacto, es gente que está muy desarraigada, que encuentra consuelo, información, y entonces mi marido y yo tuvimos la ocurrencia de agregar un foro a la página que él tenía, y así estuvimos un tiempo hasta que el año pasado lo cedió a la asociación (Carmen).

De todos modos, el hecho de que muchos de estos foros de pacientes tengan una gestión amateur, lo cual implica contar con una dedicación más puntual, estar más desprotegidos ante la entrada de publicidad engañosa, o disponer de una apariencia menos atractiva para los participantes, está provocando una disminución gradual de su uso a favor de las redes sociales generalistas. Finalmente, también cabe destacar la facilidad que supone para los pacientes poder tratar las cuestiones que hacen referencia a su salud desde las mismas plataformas que utilizan habitualmente en su día a día, y en este aspecto tanto Facebook como Whatsapp están ocupando actualmente el lugar protagonista.

\section{Discusión y consideraciones finales}

Una vez identificadas las necesidades que según el punto de vista de las personas entrevistadas pueden ser cubiertas, o parcialmente cubiertas, con el uso de tecnologías Web 2.0, a continuación nos preguntamos en qué medida la satisfacción de estas necesidades contribuye a la formación de pacientes más activos. Asimismo, en este apartado también plantearemos algunas de las medidas que puede adoptar el sistema sanitario español para acompañar esta formación de pacientes activos a través de las tecnologías Web 2.0.

Como hemos visto en el apartado dedicado a los resultados, las tres necesidades identificadas (mayor acceso a la información, mejor comuni- 
cación con los profesionales de referencia, y mayor apoyo mutuo entre pacientes) disponen de muchos puntos en común. Así, un paciente que tenga un mayor acceso a información relacionada con su enfermedad estará en mejor disposición para comunicarse de forma más fluida con sus profesionales de referencia, y a su vez, aumentará su confianza y el valor del apoyo que pueda realizar a otros pacientes que se encuentren en una situación similar a la suya. Del mismo modo, un paciente que disponga de una mejor comunicación con sus profesionales de referencia tiene más posibilidades de obtener información sanitaria de confianza relacionada con su enfermedad y de influir positivamente en los demás pacientes. Finalmente, un paciente acostumbrado a interactuar con otros pacientes sobre temas relacionados con su enfermedad tendrá mayores facilidades para asumir una actitud igualmente activa ante sus profesionales de referencia, mejorando así la comunicación con ellos, y a su vez, el hecho de interactuar con otros pacientes le propiciará un mayor acceso a información sanitaria.

Sin embargo, desde nuestro punto de vista este conjunto de factores no son suficientes para formar a pacientes plenamente activos. En primer lugar, es importante realizar un acompañamiento por parte de los profesionales sanitarios para que este mayor acceso a la información de los pacientes dé lugar, a su vez, a una mayor capacidad de autocuidarse, pasando así de la formación a la acción. No se trata únicamente de mejorar los conocimientos de los pacientes acerca de su enfermedad, sino también de mejorar su capacidad de toma de decisiones en relación a su salud y de mejorar el cumplimiento terapéutico ${ }^{12}$.

Esta distinción ha sido ampliamente abordada por Don Nutbeam ${ }^{13}$ a través del concepto Health literacy. Este autor distingue entre tres categorías de alfabetismo relacionado con la salud: la basic/functional literacy comprende la capacidad de aplicar las habilidades lectoras para entender aspectos básicos relacionados con la salud: recetas médicas, citas médicas, etc.; la communicative/interactive literacy supone una mayor capacidad social y cognitiva para procesar y utilizar nueva información, así como para utilizarla para interactuar con los demás; y la critical literacy comprende la capacidad más avanzada de analizar críticamente la información recibida y de utilizar esta información para disponer de un mayor control sobre la propia salud.

Esta clasificación pone de relieve que mayores niveles de alfabetismo relacionados con la salud otorgan una mayor capacidad de autonomía a los pacientes, a la vez que nos permite distinguir entre el acceso a la información per se y el buen manejo de esta información por parte de los pacientes. Como hemos visto en el apartado anterior, el uso de las tecnologías Web 2.0 abre nuevas posibilidades en el acceso a información en salud por parte de los pacientes. Sin embargo, esta búsqueda en ocasiones se realiza de forma indiscriminada e incluso como respuesta a la escasa información proporcionada por los profesionales sanitarios de referencia. Esto puede deberse en parte al hecho de que, si bien una gran parte de los pacientes dispone de las capacidades suficientes para realizar una búsqueda de información en internet relacionada con su enfermedad, el número de pacientes capaces de analizar esta información de forma crítica son mucho más escasos.

En este sentido, el sistema sanitario no puede dar la espalda a los cambios efectuados por parte de los pacientes en la búsqueda de información sanitaria, sino que resulta imprescindible aceptar este entorno cambiante e impulsar las medidas necesarias para dar respuesta a las inquietudes de los pacientes. Una de estas medidas puede consistir en lo que se conoce como "prescribir enlaces". Si bien son muchos los profesionales sanitarios que critican el uso de internet como fuente de información sanitaria debido al escaso control que ejercen sobre su contenido, son muy pocos los que recomiendan páginas web de referencia a sus pacientes como medida para evitar una navegación indiscriminada. Experiencias como Web Médica Acreditada, una iniciativa impulsada por el Colegio Oficial de Médicos de Barcelona y que consiste en la certificación de las páginas web de interés sanitario que cumplen con unos determinados estándares de calidad, así como portales generales de información sanitaria para pacientes como Cuidando.org o los portales de salud de las comunidades autónomas (Escuela de Pacientes, Canal Salut...), entre muchos otros, deberían ser recursos de referencia para los profesionales sanitarios, y éstos, a su vez, deberían fomentar su uso por parte de los pacientes. Asimismo, los profesionales de la salud deberían acompañar de forma más activa este proceso de formación par parte de los pacientes, destinando una parte de la consulta a resolver sus dudas y a indagar en mayor medida en sus inquietudes.

En segundo lugar, en el apartado de resultados hemos abordado la necesidad por parte de los pacientes de disponer de una mayor comunicación con sus profesionales de referencia. Sin embargo, es importante tener en cuenta que aumentar la comunicación entre los profesionales 
de la salud y los pacientes no equivale necesariamente a potenciar un rol más activo por parte de estos últimos. En este sentido, por ejemplo, muchos de los centros de salud que han puesto en funcionamiento algún tipo de herramienta para fomentar la comunicación telemática entre pacientes y profesionales de la salud, lo han hecho con el objetivo de mejorar la eficiencia de determinados trámites administrativos que pueden ser resueltos a través de internet, pero no promueven un cambio actitudinal de fondo por parte de pacientes y profesionales de la salud.

Por el contrario, consideramos que mejorar la comunicación entre pacientes y profesionales de la salud pasa por romper con la asimetría que caracteriza habitualmente esta relación y por fomentar un rol más participativo por parte de los pacientes. Como hemos visto, el uso de tecnologías Web 2.0 aplicadas al ámbito de la salud ofrece mayores posibilidades a los pacientes para influir sobre su estado de salud (mayor información, mayor interacción con otros pacientes, etc.), pero sin embargo, esta actitud cambiante por parte de algunos pacientes sigue chocando en muchas ocasiones con un modelo asistencial paternalista donde el paciente es visto simplemente como el objeto pasivo de la intervención médica ${ }^{14}$.

En este sentido, las iniciativas en favor de una medicina más participativa van ganando adeptos con el paso de los años gracias a movimientos relacionados con la toma de decisiones compartidas o la denominada "medicina basada en la afectividad", donde la comunicación entre los pacientes y los profesionales de la salud constituye una cuestión central ${ }^{15}$. Tomando prestadas las palabras de Marta Aullé, podemos afirmar que: en el proceso de toma de decisiones es tan fundamental la opinión de los médicos como la participación del enfermo. Sobre la base de una información previa, uno mismo sólo puede decidir sobre su salud si a su vez es libre de plantear preguntas para ampliar o corregir la información ${ }^{1}$.

Es desde este punto de vista que las tecnologías Web 2.0 deben entenderse como un recurso complementario a los tradicionalmente utilizados en la consulta y que permite expandir la participación de los pacientes en el sistema sanitario más allá de los horarios de consulta presencial típicamente estipulados. En definitiva, podemos afirmar que las tecnologías Web 2.0 disponen de un gran potencial para fomentar roles más activos por parte de los pacientes, pero al mismo tiempo, resulta imprescindible que todos los agentes implicados en el proceso asistencial tomen en consideración el punto de vista de los pacientes en su implementación.

\section{Colaboraciones}

M Oliver-Mora y L Iñiguez-Rueda diseñaron el estudio y supervisaron todos los aspectos de su realización. M Oliver-Mora realizó el trabajo de campo, el análisis de los resultados y la redacción del artículo. L Iñiguez-Rueda revisó críticamente el contenido intelectual del articulo y aprobó su última versión. 


\section{Referencias}

1. Allué M. El paciente inquieto. Barcelona: Edicions Bellaterra; 2013.

2. Jovell AJ, Navarro MD, Fernández L, Blancafort S. Nuevo rol del paceinte en el sistema sanitario. Atención Primaria 2006; 38(4):234-237.

3. Zapater F. Nuevos pacientes y nuevas demandes. ¿Qué hacer? In: Casajuana J, coordinador. Gestión del día a día en el Equipo de Atención Primaria. Barcelona: Sociedad Española de Medicina de Familia y Comunitaria; 2007.

4. Caballero-Uribe CV. E-pacientes y relación medico-paciente. Salud Uninorte 2013; 29(2):vi-vii.

5. Jovell AJ, Navarro MD. Profesión médica en la encrucijada: hacia un nuevo modelo de gobierno corporativo y de contrato social. Madrid: Fundación Alternativas; 2006.

6. Oliver-Mora M, Iñiguez-Rueda L. Identificación de experiencias de Salud 2.0 en el ámbito de la atención primaria en España. Índex de enfermería. No prelo 2017.

7. Adahl K. Transparency of Critical Information for Patient Empowerment in eHealth. Karlskrona: Blekinge Institute of Technology; 2007.

8. Eysenbach G. What is e-health? Journal of Medical Internet Research (edición digital) 2001. [acceso 2014 oct 2]. Disponible en: http://www.jmir.org/2001/2/e20/

9. Hughes B, Joshi I, Wareham J. Health 2.0 and Medicine 2.0: Tensions and Controversies in the Field. J Med Internet Res 2008; 3(10):e23. [acceso 2015 feb 16]. Disponible en: http://www.jmir.org/2008/3/e23/

10. Pagliari C, Sloan D, Gregor P, Sullivan F, Detmer D, Kahan JP, Oortwijn W, MacGillivray S. What is eHealth (4): A Scoping Exercise to Map de Field. J Med Internet Res (edición digital) 2005; 7(1). [acceso 2014 oct 2]. Disponible en: http://www.jmir.org/2005/1/e9/

11. Vázquez F. El análisis de contenido categorial: el análisis temático. Barcelona: Universidad Autónoma de Barcelona; 1994.

12. Millaruelo JM. Importancia de la implicación del paciente en el autocontrol de su enfermedad: paciente experto. Importancia de las nuevas tecnologías como soporte al paciente autónomo. Atención Primaria 2010; 42(Supl. 1):41-47.

13. Nutbeam D. Health literacy as a public goal: a challange for contemporany public health education and communication strategies into the 21 st century. Health Promotion International 2000; 3(15):259-267.

14. Adahl K. On decision support in participatory medicine supporting health care empowerment. Karlskrona: Blekinge Institute of Technology; 2012.

15. Jovell AJ. Medicina basada en la afectividad. Medicina Clínica 1999; 113:173-175. 